\title{
Operation of gas proportional scintillation counters in a low charge multiplication regime
}

\author{
F.I.G.M. Borges*, J.M.F. dos Santos, T.H.V.T. Dias, F.P. Santos, \\ P.J.B.M. Rachinhas, C.A.N. Conde \\ Departamento de Física, Universidade de Coimbra, P-3000 Coimbra, Portugal
}

\begin{abstract}
The operation of a xenon filled gas proportional scintillation counter under low charge multiplication gains is discussed. It is shown that the best energy resolution for X-rays is obtained for a reduced electric field, $E / p$, of about $8 \mathrm{~V} \mathrm{~cm}^{-1}$ Torr $^{-1}$, leading to a charge gain (for inter-grid distance of $5 \mathrm{~mm}$ ) of about 1.05, as calculated by a Monte Carlo simulation method. A discussion is presented concerning the dependence of the $E / p$ values for better detector performance, on the inter-grid distance. (C) 1999 Elsevier Science B.V. All rights reserved.
\end{abstract}

\section{Introduction}

The gas proportional scintillation counter (GPSC) is a noble gas X-ray detector that delivers superior performance when compared with other gas detectors, since it combines a better energy resolution $[1,2]$ with a high counting rate capability due to the absence of space-charge effects. In this type of detector, amplification of the primary ionization charge is achieved by the production of secondary scintillation (VUV electroluminescence), which results in reduced statistical fluctuations when compared to the charge avalanche process used in gas detectors of the proportional counter (PC) type. Therefore, GPSCs are usually operated

\footnotetext{
* Corresponding author.
}

with scintillation electric fields just below the charge multiplication threshold. This threshold has been assumed to occur in xenon at a reduced electric field of $E / p \sim 6 \mathrm{~V} \mathrm{~cm}^{-1} \mathrm{Torr}^{-1}$ [1-3], where $E$ is the electric field strength and $p$ the gas pressure.

In this work, we investigate the performance of a xenon filled GPSC operating under a low charge multiplication regime, measuring the pulseheight distributions and the energy resolutions for 2.6, 5.9, 14 and $22 \mathrm{keV}$ X-rays, using reduced electric fields in the scintillation region between 2 and $11 \mathrm{~V} \mathrm{~cm}^{-1} \mathrm{Torr}^{-1}$. The experimental data are compared with the results obtained with a detailed Monte Carlo simulation for the drift of electrons in xenon [4], and an assessment is made of the extent to which charge multiplication can be allowed without deteriorating the energy resolution. 


\section{Rationale}

The energy resolution of a conventional proportional counter is limited by the statistical fluctuations in the primary ionization and in the electron multiplication processes, and can be given by $[5]$

$R=2.35 \sqrt{\frac{(F+f)}{\bar{N}}}$,

where $\bar{N}$ is the average number of primary (subionization) electrons produced per X-ray photon, $F=\sigma_{N}^{2} / \bar{N}$ is the relative variance of $N(F$ is usually called the Fano factor) and $f=\left(\sigma_{\mathrm{G}} / \bar{G}\right)^{2}$ is a measure of the fluctuations in the electron multiplication gain $G$. For large enough gains, the parameter $f$ is independent of $G$, and is larger than $F$ (e.g. $f=0.6$ and $F=0.2$ for xenon $[5,6]$ ), thereby the energy resolution of a $\mathrm{PC}$ is dominated by $f$.

On the other hand, the energy resolution of a conventional GPSC operating just below charge multiplication is determined by the statistical fluctuations in the primary ionization processes, in the production of VUV scintillation photons and in the photonsensor performance, and can be given by $[7,8]$

$R=\sqrt{\frac{F}{\bar{N}}+\frac{1}{\bar{N}} \cdot\left(\frac{J}{\bar{N}_{\mathrm{s}}}\right)^{2}+\frac{1}{\bar{N}_{\mathrm{e}}}\left(1+\left(\frac{\sigma_{q}}{\bar{G}_{q}}\right)^{2}\right)}$,

where $\bar{N}_{\mathrm{s}}$ is the average number of scintillation photons produced per primary electron, $J=\sigma_{\mathrm{s}}^{2} / \bar{N}_{\mathrm{s}}$ is the relative variance of $N_{\mathrm{s}}, \bar{N}_{\mathrm{e}}$ is the average number of photoelectrons produced in the photosensor per incident X-ray photon, and $\left(\sigma_{q} / \bar{G}_{q}\right)^{2}$ is a measure of the fluctuations in the electron multiplication gain in the photosensor. Other parameters are defined as in Eq. (1).

The most common photosensor used in a GPSC is the photomultiplier tube, for which $\left(\sigma_{q} / \bar{G}_{q}\right)^{2} \approx 1$ [7]. Moreover, since $J \ll F$ and $\bar{N}_{\mathrm{s}}>100$ [8], the second term of Eq. (2) can be neglected, and the energy resolution of a GPSC can be approximated by

$$
R=2.35 \sqrt{\frac{F}{\bar{N}}+\frac{2}{\bar{N}_{\mathrm{e}}}} .
$$

The energy resolution of a GPSC is thus dominated by the Fano factor $F$. However, if electron multiplication is allowed in the GPSC to $F$ is added a term, $f$, that corresponds to the statistical fluctuations of the charge multiplication and $R$ deteriorates approaching the case of standard proportional counters [9]

$R=2.35 \sqrt{\frac{(F+f)}{\bar{N}}+\frac{2}{\bar{N}_{\mathrm{e}}}}$.

It has been pointed out $[10,11]$ that it could be of interest to operate a GPSC under a low multiplication charge regime since then $f$ is much smaller than $F$ [12]. Indeed, a small increase in the first term of $R$ may be compensated by a reduction in the second term, caused by an increased scintillation yield and $\bar{N}_{\mathrm{e}}$. This may eventually result in a better overall energy resolution, but this was never actually confirmed.

We now revisit the earlier assumptions of Refs. $[10,11]$ and investigate the extent to which charge multiplication can be allowed in the operation of a GPSC in order to achieve better performance. The maximization of the reduced electric field and scintillation yield can be important in applications such as soft X-ray driftless detectors (to reduce primary electron loss to the detector window $[3,7,13])$ and in cases where low scintillation output occurs [14]. Improvement in position resolution may also be achieved [10].

\section{Experimental set-up and results}

The GPSC we used to obtain the experimental results was developed in our laboratory for energy dispersive X-ray analysis applications and is depicted schematically in Fig. 1. The detector is a non-focused uniform field GPSC with a $0.54 \mathrm{~cm}$ scintillation region instead of the typical $1 \mathrm{~cm}$ scintillation region described in Refs. [15,16]. This scintillation depth enables us to reach higher $E / p$ values at lower applied voltages, since we were limited by electric breakdown. The second grid of the detector was vacuum deposited onto the surface of the quartz window of the EMI D676QB photomultiplier tube used. The radiation entrance 


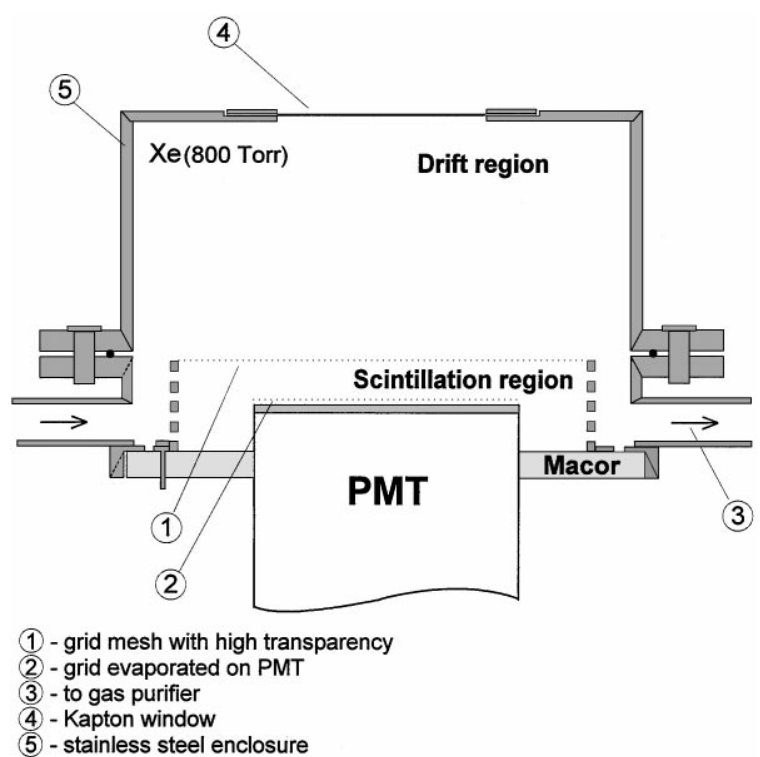

Fig. 1. Schematic of the GPSC used in this work.

window was a $50 \mu \mathrm{m}$ thick aluminized Kapton film. The detector was filled with high-purity xenon at 800 Torr, continuously purified during operation.

The measured pulse-height distributions were fitted to gaussian functions superimposed on a linear background using the GRIDLS method [17], from which the centroid and the full width at half maximum were taken.

We investigated the performance of the GPSC, measuring the energy resolution and centroid position of the pulse-height distributions for different $\mathrm{X}$-ray energies $(2.6,5.9,14$ and $22 \mathrm{keV})$ as a function of the reduced electric field $E / p$ applied in the scintillation region, in the $2-11 \mathrm{~V} \mathrm{~cm}^{-1} \mathrm{Torr}^{-1}$ range. Note that the centroid position is proportional to the scintillation yield $Y / p$ (number of VUV scintillation photons per electron per $\mathrm{cm}$ per Torr). The reduced electric field in the drift region was kept constant at about $0.3 \mathrm{~V} \mathrm{~cm}^{-1} \mathrm{Torr}^{-1}$.

In Fig. $2 \mathrm{a}$ and $\mathrm{b}$ we present the reduced scintillation yield $Y / p$ and the detector energy resolution $R$ as a function of $E / p$ in the scintillation region for 5.9 and $22 \mathrm{keV}$ incident $\mathrm{X}$-ray photons $(1 \mathrm{~mm}$ collimated). As it can be observed, the scintillation yields, with a threshold at about $1 \mathrm{~V} \mathrm{~cm}^{-1} \mathrm{Torr}^{-1}$, follow a linear trend until an $E / p$ of $8 \mathrm{~V} \mathrm{~cm}^{-1} \mathrm{Torr}^{-1}$ in both cases. Above this value,

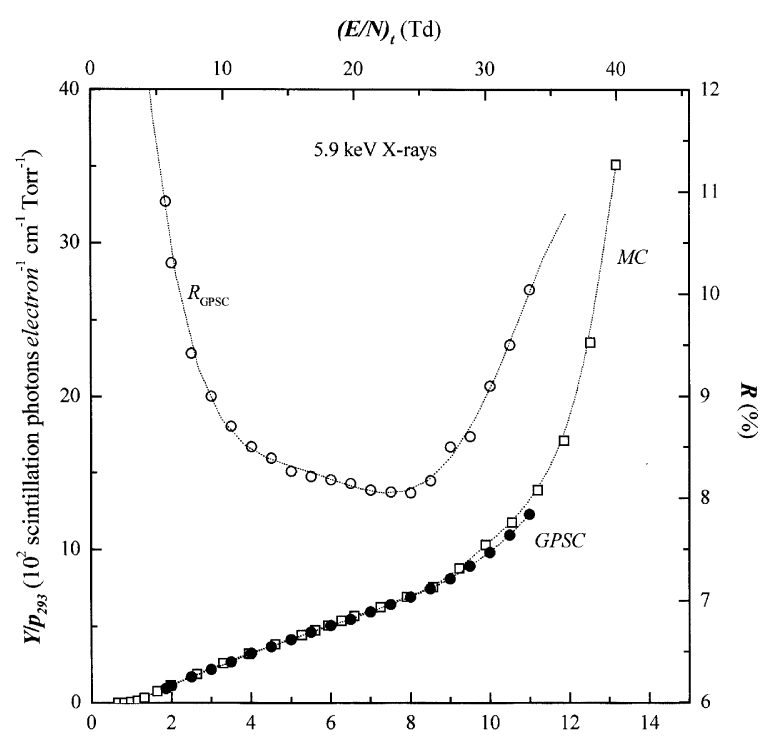

(a)

$$
(E / p)_{293}\left(\mathrm{~V} \mathrm{~cm}^{-1} \operatorname{Torr}^{-1}\right)
$$

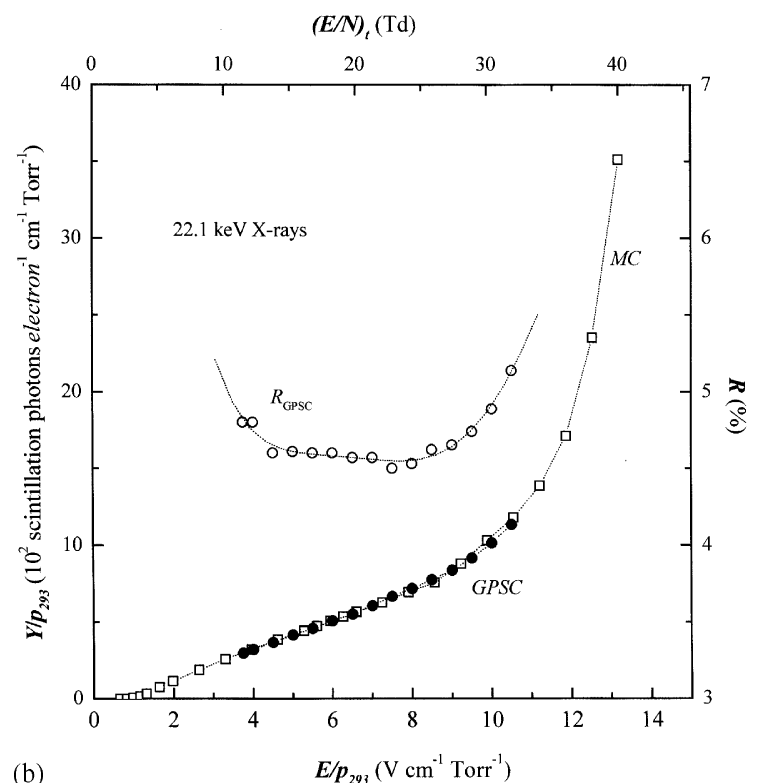

Fig. 2. Reduced scintillation yield ( $\square-$ Monte Carlo results; - experimental results) and detector energy resolution $(\bigcirc)$ as a function of reduced electric field in the scintillation region for: (a) $5.9 \mathrm{keV} \mathrm{X}$-rays and (b) $22.1 \mathrm{keV} \mathrm{X}$-rays. The experimental values have been normalized to the calculated Monte Carlo value at $E / p=6 \mathrm{~V} \mathrm{~cm}^{-1} \mathrm{Torr}^{-1}$. 
the data departs from the linear behaviour, reflecting the exponential increase in the number of VUV scintillation photons when charge multiplication takes place. The best energy resolution was obtained for an $E / p$ value of about $8 \mathrm{~V} \mathrm{~cm}^{-1} \mathrm{Torr}^{-1}$, well above the ionization threshold of $6 \mathrm{~V} \mathrm{~cm}^{-1}$ Torr ${ }^{-1}$ and above the $E / p$ in the $5-6 \mathrm{~V} \mathrm{~cm}^{-1}$ Torr $^{-1}$ region usually considered for the optimum operation of xenon filled GPSCs [1-3]. The same trend was observed for the other X-ray energies studied.

\section{Monte Carlo calculations and discussion}

The experimental scintillation yield results for 5.9 and $22 \mathrm{keV}$ X-rays are compared in Fig. 2 with the results for a detailed Monte Carlo simulation. A full description of the Monte Carlo model and results is made in Refs. [4,18]. As it is observed in Fig. 2, a very good agreement between experimental and simulation results is found. The experimental values in Fig. 2 have been normalized to the absolute Monte Carlo value at $E / p=$ $6 \mathrm{~V} \mathrm{~cm}^{-1} \mathrm{Torr}^{-1}$.

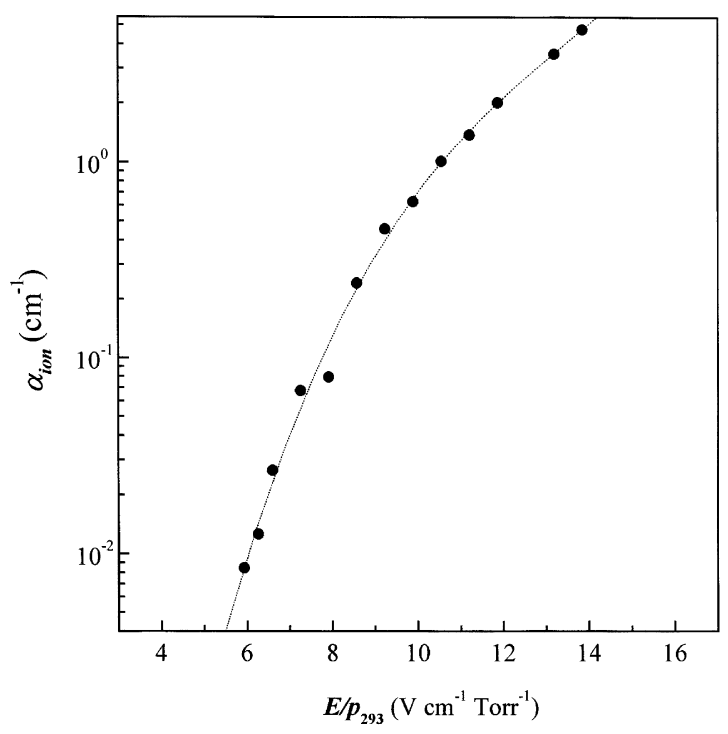

Fig. 3. Monte Carlo calculated first Townsend coefficient, $\alpha$, as a function of reduced electric field in the scintillation region, for xenon at 800 Torr.
To evaluate the extent to which ionization takes place for the range of reduced electric fields in the experiments, we use the Monte Carlo calculated first Townsend ionization coefficient, $\alpha$, represented in Fig. 3. The Monte Carlo results for $\alpha$ indicate that the xenon ionization threshold occurs at $E / p \sim 6 \mathrm{~V} \mathrm{~cm}^{-1} \mathrm{Torr}^{-1}$ and that the value for $\alpha$ at $E / p=8 \mathrm{~V} \mathrm{~cm}^{-1} \mathrm{Torr}^{-1}$ is about $0.1 \mathrm{~cm}^{-1}$ $(p=800$ Torr $)$. To this $\alpha$ value corresponds a charge gain of about 1.05 across the $0.54 \mathrm{~cm}$ scintillation depth, well below the gain values pointed out in Ref. [11] (below about 10).

If we assume that a charge gain of 1.05 is the maximum that can be allowed across the scintillation region before energy resolution starts to deteriorate, then the optimum $E / p$ depends on the depth $d$ of the detector scintillation region. In Fig. 4 we represent, as a function of the depth $d$, the $E / p$ value required to obtain a 1.05 charge gain across $d$. These results are deduced from the Monte Carlo $\alpha$ calculations. Fig. 4 shows that a charge gain of 1.05 is achieved at $E / p$ values that can be as high as $8.7 \mathrm{~V} \mathrm{~cm}^{-1}$ Torr $^{-1}$ for $d=0.3 \mathrm{~cm}$ (this value of $d$ is used in [14]), $6.9 \mathrm{~V} \mathrm{~cm}^{-1} \mathrm{Torr}^{-1}$ for $d=1.5 \mathrm{~cm} \mathrm{[2]}$ and only $6.5 \mathrm{~V} \mathrm{~cm}^{-1}$ Torr $^{-1}$ for $d=4 \mathrm{~cm}$ [19].

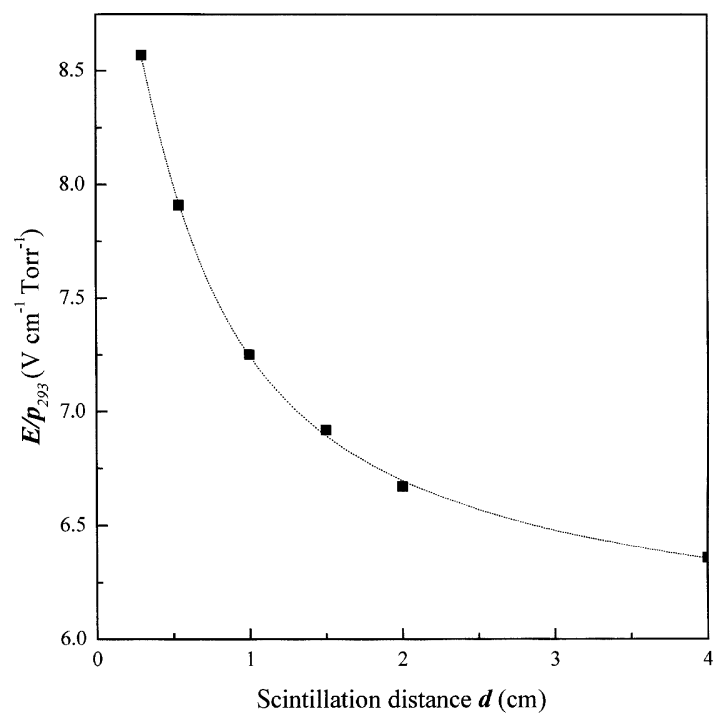

Fig. 4. Reduced electric field in the scintillation region required to obtain a 1.05 charge gain as a function of the scintillation region depth. 


\section{Conclusions}

The operation of a xenon GPSC in a low charge multiplication regime was discussed. It was found that for a scintillation region depth of $0.54 \mathrm{~cm}$ the best GPSC energy resolution is achieved for an $E / p$ value of $8 \mathrm{~V} \mathrm{~cm}^{-1} \mathrm{Torr}^{-1}$, which is well above the 5-6 $\mathrm{V} \mathrm{cm}^{-1} \mathrm{Torr}^{-1}$ values usually considered for optimum GPSC operation. The results of a Monte Carlo simulation indicate that the $E / p=$ $8 \mathrm{~V} \mathrm{~cm}^{-1} \mathrm{Torr}^{-1}$ field applied across the $0.54 \mathrm{~cm}$ scintillation region yields a charge gain of 1.05 . The Monte Carlo results also indicate that for GPSC scintillation depths increasing from 0.3 to $4 \mathrm{~cm}$, an optimum charge gain of 1.05 will be achieved at $E / p$ values in the $8.7-6.5 \mathrm{~V} \mathrm{~cm}^{-1} \mathrm{Torr}^{-1}$ range.

\section{Acknowledgements}

This work was carried out in the Atomic and Nuclear Instrumentation Group of the Instrumentation Centre (Unit 217/94) of the Physics Department, University of Coimbra. This work was supported by Project CERN/P/FAE/1163/97. P.J.B.M. Rachinhas was supported through the JNICT grant BD-2652/94. Travel support is acknowledged from Fundação Luso-Americana para o Desenvolvimento (FLAD), Lisbon.

\section{References}

[1] C.A.N. Conde, L. Requicha Ferreira, M. Fátima A. Ferreira, IEEE Trans. Nucl. Sci. 24 (1977) 221.
[2] A. Peacock, R.D. Andresen, E.-A. Leimann, A.E. Long, G. Manzo, B.G. Taylor, Nucl. Instr. and Meth. 169 (1980) 613.

[3] M. Barbera, A. Collura, F. Favata, G. Inzerillo, F. Mirabello, Proc. SPIE - EUV, X-ray, and Gamma-ray Instrumentation for Astronomy V, 27-28 July 1994, San Diego, CA, vol. 2280, 1994.

[4] T.H.V.T. Dias, F.P. Santos, A.D. Stauffer, C.A.N. Conde, Phys. Rev. A 48 (1993) 2887.

[5] G.F. Knoll, Radiation Detection and Measurement, 2nd ed., Wiley, New York, 1989, pp. 160-198.

[6] P.J.B.M. Rachinhas, T.H.V.T. Dias, A.D. Stauffer, F.P. Santos, C.A.N. Conde, IEEE Trans. Nucl. Sci. 43 (1996) 2399.

[7] D.G. Simons, P.A.J. De Korte, Nucl. Instr. and Meth. A 277 (1989) 642.

[8] T.H.V.T. Dias, Simulação do Transporte de Electrões em Gases Raros, Ph.D. Thesis, Universidade de Coimbra, 1986.

[9] S.P. Garg, R.C. Sharma, Nucl. Instr. and Meth. 222 (1984) 574.

[10] A.J.P.L. Policarpo, M.A.F. Alves, M.C.M. dos Santos, M.J.T. Carvalho, Nucl. Instr. and Meth. 102 (1972) 337.

[11] A.J.P.L. Policarpo, Space Sci. Instr. 3 (1977) 77.

[12] G.D. Alkazov, Nucl. Instr. and Meth. 89 (1970) 155.

[13] T.H.V.T. Dias, F.P. Santos, A.D. Stauffer, C.A.N. Conde, Phys. Rev. A 46 (1992) 237.

[14] V. Dangendorf, H. Schmidt-Bocking, Proc. SPIE - EUV, $\mathrm{X}$-ray, and Gamma-ray Instrumentation for Astronomy V, 6-11 August 1989, San Diego, CA, vol. 1159, 1989, p. 192.

[15] J.M.F. dos Santos, A.C.S.S.M. Bento, C.A.N. Conde, X-ray Spectrom. 22 (1993) 328.

[16] J.M.F. dos Santos, J.F.C.A. Veloso, R.E. Morgado, C.A.N. Conde, Nucl. Instr. and Meth. A 353 (1994) 195.

[17] P.R. Bevington, Data Reduction and Error Analysis for the Physical Sciences, McGraw-Hill, New York, 1969, pp. 208-214.

[18] F.P. Santos, T.H.V.T. Dias, P.J.B.M. Rachinhas, A.D. Stauffer, C.A.N. Conde, IEEE Trans. Nucl. Sci. 45 (April 1998).

[19] A. Smith, A. Peacock, T.Z. Kowalski, IEEE Trans. Nucl. Sci. NS-34 (1987) 57. 